\title{
Triple solutions for a damped impulsive differential equation
}

\author{
Jian Liu ${ }^{1 *}$, Zengqin Zhao ${ }^{2}$, Wenguang $\mathrm{Yu}^{3^{*}}$ and Tongqian Zhang ${ }^{4}$
}

\section{"Correspondence:}

sxkj163@163.com;

yuwg@sdufe.edu.cn

${ }^{1}$ School of Mathematics and

Quantitative Economics, Shandong

University of Finance and

Economics, Jinan, P.R. China

${ }^{3}$ School of Insurance, Shandong

University of Finance and

Economics, Jinan, P.R. China

Full list of author information is

available at the end of the article

\section{Springer}

\begin{abstract}
In this paper, we aim to obtain the existence of at least three classical solutions for nonlinear impulsive problems neither adding superlinear or local sublinear conditions to nonlinear term at zero nor adding superlinear assumptions to nonlinear term at infinity. Our approach relies on variational methods, more specifically, on a three critical points theorem.
\end{abstract}

MSC: $34 \mathrm{~B} 15 ; 35 \mathrm{~B} 38$

Keywords: Triple solutions; Damped term; Impulsive effects

\section{Introduction}

Recently, a great deal of work has been done in dealing with the existence of solutions for impulsive differential equations, for example, [1-12] and the references therein.

Nieto initially introduced a new approach via variational methods to obtain the existence of a weak solution to linear impulsive problems in [1] by the Lax-Milgram theorem, and also obtained at least one weak solution to nonlinear problems via Mountain Pass Theorem.

Moreover, Nieto in [2], by using the Lax-Milgram theorem studied the existence of a unique weak solution for a differential equations with impulses and with a discontinuous damped term.

In [3], by exploiting critical point theory and variational methods, we obtained the existence of one and infinity many solutions for some impulsive boundary value problems with a parameter when the nonlinear term satisfied the Ambrosetti-Rabinowitz type superlinear condition.

In [4], we considered impulsive differential equations with small non-autonomous perturbations via variational methods and a three critical points theorem. The existence of at least three classical solutions was obtained when the primitive function of nonlinearity $f(t, u)$ satisfied some superlinear conditions at infinity.

In contrast to [3] and [4], the aim of this paper is to deal with the existence of at least three classical solutions of the following damped impulsive differential equations:

$$
\left\{\begin{array}{l}
-u^{\prime \prime}(t)+u(t)+g(t) u^{\prime}(t)=\lambda f(t, u) \quad \text { a.e. } t \in[0, T] \\
\Delta u^{\prime}\left(t_{i}\right)=I_{i}\left(u\left(t_{i}\right)\right) \quad i=1,2, \ldots, p \\
u(0)=u(T)=0
\end{array}\right.
$$

(c) The Author(s) 2019. This article is distributed under the terms of the Creative Commons Attribution 4.0 International License (http://creativecommons.org/licenses/by/4.0/), which permits unrestricted use, distribution, and reproduction in any medium, provided you give appropriate credit to the original author(s) and the source, provide a link to the Creative Commons license, and indicate if changes were made. 
where $\lambda$ is a parameter, $T>0, g \in C[0, T], f \in C([0, T] \times \mathbb{R}, \mathbb{R})$ and $I_{i}: \mathbb{R} \rightarrow \mathbb{R}(i=1,2, \ldots, p)$ are continuous, $0=t_{0}<t_{1}<\cdots<t_{p}<t_{p+1}=T, \Delta u^{\prime}\left(t_{i}\right)=u^{\prime}\left(t_{i}^{+}\right)-u^{\prime}\left(t_{i}^{-}\right)=\lim _{t \rightarrow t_{i}^{+}} u^{\prime}(t)-$ $\lim _{t \rightarrow t_{i}^{-}} u^{\prime}(t)$, without assuming asymptotic conditions neither at zero nor at infinity on the nonlinear term. Our main tools are variational methods and a three critical points theorem by Bonanno and Marano.

We need the following assumptions:

$\left(H_{1}\right) I_{i}(u)$ are nondecreasing, and $I_{i}(u) u \geq 0$ for any $u \in \mathbb{R}$.

$\left(H_{2}\right)$ There exist positive constants $C_{0}, a, l$ with $l<2$, and a function $b \in L^{1}[0, T]$ such that for all $|u|>C_{0}$ and $t \in[0, T]$

$$
|F(t, u)| \leq b(t)\left(a+|u|^{l}\right)
$$

where $F(t, u)=\int_{0}^{u} f(t, s) d s$.

It is worth pointing out that conditions $\left(H_{2}\right)$ and $\left(H_{4}\right)$ in Theorem 3.1 of this paper are not local conditions at zero, thus we neither add any superlinear or sublinear assumptions to the nonlinear term at zero nor add superlinear assumptions to the nonlinear term at infinity.

We would also like to mention the work of Candito, Carl and Livrea in [13-15], in which non-coercive differential problems involving a positive parameter $\lambda$ were investigated. Authors presented novel variational approaches to obtain multiplicity, regularity, and a priori estimates of solutions by assuming certain growth conditions on the nonlinearity prescribed only near zero.

The rest of the paper is organized as follows: In Sect. 2, we give variational structure. In Sect. 3, we formulate and prove our results, and an example is presented to illustrate our results.

\section{Variational structure}

Our aim is to get the existence of at least three classical solutions for nonlinear impulsive problems by using variational methods. The main tool we used is the following three critical points theorem obtained in [16] or [17].

Theorem 2.1 ([16, 17]) Let $X$ be a reflexive real Banach space, $\varphi: X \rightarrow \mathbb{R}$ a sequentially weakly lower semicontinuous, coercive and continuously Gâteaux differentiable functional whose Gâteaux derivative admits a continuous inverse on $X^{*}$, and let $\phi: X \rightarrow \mathbb{R}$ be a sequentially weakly upper semicontinuous, continuously Gâteaux differentiable functional whose Gâteaux derivative is compact. Assume that there exist $r \in \mathbb{R}$ and $x_{0}, \bar{x} \in X$, with $\varphi\left(x_{0}\right)<r<\varphi(\bar{x})$, and $\phi\left(x_{0}\right)=0$ such that

(i) $\sup _{\varphi(x) \leq r} \phi(x)<\left(r-\varphi\left(x_{0}\right)\right) \frac{\phi(\bar{x})}{\varphi(\bar{x})-\varphi\left(x_{0}\right)}$,

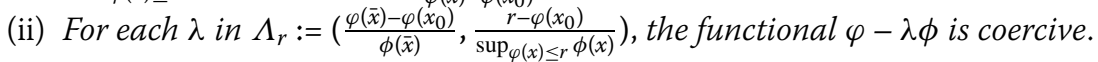

Then, for each $\lambda \in \Lambda_{r}$ the functional $\varphi-\bar{\lambda} \phi$ has at least three distinct critical points in $X$.

Let $\alpha=\min _{t \in[0, T]} e^{G(t)}, \beta=\max _{t \in[0, T]} e^{G(t)}$, and $G(t)=-\int_{0}^{t} g(s) d s$.

Denote by $H:=H_{0}^{1}(0, T)=\left\{u:[0, T] \rightarrow \mathbb{R} \mid u\right.$ is absolutely continuous, $u^{\prime} \in L^{2}(0, T)$, $u(0)=u(T)=0\}$ the Sobolev space with the inner product and induced norm given by

$$
(u, v)_{H}=\int_{0}^{T} e^{G(t)}\left(u(t) v(t)+u^{\prime}(t) v^{\prime}(t)\right) d t
$$




$$
\|u\|_{H}=\left(\int_{0}^{T} e^{G(t)}\left(u^{\prime}(t)^{2}+u(t)^{2}\right) d t\right)^{1 / 2} .
$$

It is well known that $H$ is a reflexive Banach space [18, p. 7].

Consider the functional $\varphi(u)-\lambda \phi(u): H \rightarrow \mathbb{R}$, where

$$
\begin{aligned}
& \varphi(u)=\frac{1}{2}\|u\|_{H}^{2}+\sum_{i=1}^{p} e^{G\left(t_{i}\right)} \int_{0}^{u\left(t_{i}\right)} I_{i}(t) d t, \\
& \phi(u)=\int_{0}^{T} e^{G(t)}(F(t, u(t)) d t .
\end{aligned}
$$

In view of the continuity of $f$ and $I_{i}(i=1,2, \ldots, p)$, we obtain that $\varphi$ and $\phi$ are Gateaux differentiable and

$$
\begin{aligned}
(\varphi-\lambda \phi)^{\prime}(u) v= & \int_{0}^{T} e^{G(t)} u^{\prime}(t) v^{\prime}(t) d t+\int_{0}^{T} e^{G(t)} u(t) v(t) d t \\
& +\sum_{i=1}^{p} e^{G\left(t_{i}\right)} I_{i}\left(u\left(t_{i}\right)\right) v\left(t_{i}\right)-\lambda \int_{0}^{T} e^{G(t)} f(t, u(t)) v(t) d t
\end{aligned}
$$

for any $v \in H$. Hence, a critical point of the functional $\varphi-\lambda \phi$ gives us a weak solution of problem (1.1).

Definition 2.2 We say that $u \in H$ is a weak solution of problem (1.1) if

$$
\begin{aligned}
& \int_{0}^{T} e^{G(t)} u^{\prime}(t) v^{\prime}(t) d t+\int_{0}^{T} e^{G(t)} u(t) v(t) d t+\sum_{i=1}^{p} e^{G\left(t_{i}\right)} I_{i}\left(u\left(t_{i}\right)\right) v\left(t_{i}\right) \\
& -\lambda \int_{0}^{T} e^{G(t)} f(t, u(t)) v(t) d t=0
\end{aligned}
$$

holds for any $v \in H$.

By the same calculations as in [6], we get the following lemma.

Lemma 2.3 ([6]) If $u \in H$ is a weak solution of problem (1.1), then $u$ is a classical solution of problem (1.1), and there exists $M>0$ such that $\|u\|_{\infty} \leq M\|u\|_{H}$, where $u \in H,\|u\|_{\infty}=$ $\max _{t \in[0, T]}|u(t)|$.

Definition 2.4 ([18]) Let $E$ be a Banach space. A functional $\varphi: E \rightarrow \mathbb{R}$ is said to be sequentially weakly lower semicontinuous if $\liminf _{n \rightarrow+\infty} \varphi\left(x_{n}\right) \geq \varphi(x)$ as $x_{n} \rightarrow x$ in $E$.

Lemma 2.5 Assume that $\left(H_{1}\right)$ holds. Then the functional $\varphi$ is sequentially weakly lower semicontinuous, coercive, and its derivative admits a continuous inverse on $H^{*}$.

Proof Firstly, we prove that $\varphi$ is sequentially weakly lower semicontinuous.

Let $u_{n} \rightarrow u$ in $H$, then one has $\|u\| \leq \liminf _{n \rightarrow \infty}\left\|u_{n}\right\|$, and $u_{n} \rightrightarrows u$ in $[0, T]$ when $n \rightarrow \infty$, hence

$$
\liminf _{n \rightarrow \infty} \varphi\left(u_{n}\right)=\liminf _{n \rightarrow \infty}\left(\frac{1}{2}\left\|u_{n}\right\|_{H}^{2}+\sum_{i=1}^{p} e^{G\left(t_{i}\right)} \int_{0}^{u_{n}\left(t_{i}\right)} I_{i}(t) d t\right)
$$




$$
\begin{aligned}
& \geq \frac{1}{2}\|u\|_{H}^{2}+\sum_{i=1}^{p} e^{G\left(t_{i}\right)} \int_{0}^{u\left(t_{i}\right)} I_{i}(t) d t \\
& =\varphi(u) .
\end{aligned}
$$

Thus, by Definition 2.4, $\varphi$ is sequentially weakly lower semicontinuous.

Moreover, condition $\left(H_{1}\right)$ implies that $\sum_{i=1}^{p} \int_{0}^{u\left(t_{i}\right)} I_{i}(t) d t \geq 0$, thus

$$
\varphi(u)=\frac{1}{2}\|u\|_{H}^{2}+\sum_{i=1}^{p} e^{G\left(t_{i}\right)} \int_{0}^{u\left(t_{i}\right)} I_{i}(t) d t \geq \frac{1}{2}\|u\|_{H}^{2}
$$

so the functional $\varphi$ is coercive.

Secondly, we prove that $\varphi^{\prime}$ admits a continuous inverse on $H^{*}$.

For any $u \in H \backslash\{0\}$, one has

$$
\begin{aligned}
\left\langle\varphi^{\prime}(u), u\right\rangle= & \int_{0}^{T} e^{G(t)} u^{\prime}(t) u^{\prime}(t) d t+\int_{0}^{T} e^{G(t)} u(t) u(t) d t \\
& +\sum_{i=1}^{p} e^{G\left(t_{i}\right)} I_{i}\left(u\left(t_{i}\right)\right) u\left(t_{i}\right) \\
\geq & \|u\|_{H}^{2},
\end{aligned}
$$

thus

$$
\liminf _{\|u\|_{H} \rightarrow \infty} \frac{\left\langle\varphi^{\prime}(u), u\right\rangle}{\|u\|_{H}}=+\infty
$$

and so $\varphi^{\prime}$ is coercive.

In view of the assumption that $I_{i}(u)(i=1,2, \ldots, p)$ are nondecreasing, for any $u, v \in H$, one has

$$
\begin{aligned}
\left\langle\varphi^{\prime}(u)-\varphi^{\prime}(v), u-v\right\rangle= & \int_{0}^{T} e^{G(t)}\left(u^{\prime}(t)-v^{\prime}(t)\right)\left(u^{\prime}(t)-v^{\prime}(t)\right) d t \\
& +\int_{0}^{T} e^{G(t)}(u(t)-v(t))(u(t)-v(t)) d t \\
& +\sum_{i=1}^{p} e^{G\left(t_{i}\right)} I_{i}\left(u\left(t_{i}\right)-v\left(t_{i}\right)\right)\left(u\left(t_{i}\right)-v\left(t_{i}\right)\right) \\
\geq & \|u-v\|_{H}^{2},
\end{aligned}
$$

thus we have that $\varphi^{\prime}$ is uniformly monotone. Taking account of [19, Theorem 26], we get that $\left(\varphi^{\prime}\right)^{-1}$ exists and is continuous on $H^{*}$.

Lemma 2.6 The functional $\phi$ is a sequentially weakly upper semicontinuous continuously Gâteaux differentiable functional and its derivative is compact. 
Proof Let $\left\{u_{n}\right\} \in H$ satisfying $u_{n} \rightarrow u \in H$, then $u_{n} \rightrightarrows u$ in $[0, T]$ as $n \rightarrow+\infty$. By using the Reverse Fatou Lemma, one has

$$
\begin{aligned}
\limsup _{n \rightarrow \infty} \phi\left(u_{n}\right) & =\limsup _{n \rightarrow \infty} \int_{0}^{T} e^{G(t)} F\left(t, u_{n}(t)\right) d t \\
& \leq \lim _{n \rightarrow \infty} \int_{0}^{T} e^{G(t)} \sup F\left(t, u_{n}(t)\right) d t \\
& =\int_{0}^{T} e^{G(t)} F(t, u(t)) d t=\phi(u) .
\end{aligned}
$$

Thus, $\phi$ is sequentially weakly upper semicontinuous. Now, we prove that $\phi^{\prime}$ is strongly continuous on $H$. Noting that $f(t, u)$ is continuous on $u, f\left(t, u_{n}\right) \rightarrow f(t, u)$ as $n \rightarrow+\infty$, and then

$$
\lim _{n \rightarrow \infty} \int_{0}^{T} f\left(t, u_{n}(t)\right) v d t=\int_{0}^{T} f(t, u(t)) v d t, \quad \forall v \in H
$$

Thus we get that $\phi^{\prime}$ is strongly continuous on $H$, which implies that $\phi^{\prime}$ is compact by [19, Proposition 26.2]. In addition, $\phi^{\prime}$ is continuous taking into account that it is strongly continuous.

\section{Main results}

Without adding any superlinear or sublinear local condition on the potential $F$ at zero, we get the following theorems.

Theorem 3.1 Suppose that $\left(H_{1}\right)$ and $\left(H_{2}\right)$ hold, and there exist positive constants $c, d$ such that

$$
\begin{aligned}
& \left(H_{3}\right) \frac{c^{2}}{M^{2}}<d^{2}\left\|e^{G(t)}\right\|_{L^{1}}+2 \sum_{i=1}^{p} e^{G\left(t_{i}\right)} \int_{0}^{d} I_{i}(t) d t \\
& \left(H_{4}\right) \frac{M^{2} \int_{0}^{T} \max _{|s| \leq c} F(t, s) d t}{c^{2}}<\frac{\int_{0}^{T} e^{G(t)} F(t, d) d t}{d^{2}\left\|e^{G(t)}\right\|_{L^{1}}+2 \sum_{i=1}^{p} e^{G\left(t_{i}\right)} \int_{0}^{d} I_{i}(t) d t},
\end{aligned}
$$

then for any $\lambda \in \Lambda_{r}:=\left(\frac{d^{2}\left\|e^{G(t)}\right\|_{L^{1}}+2 \sum_{i=1}^{p} e^{G\left(t_{i}\right)} \int_{0}^{d} I_{i}(t) d t}{2 \int_{0}^{T} e^{G(t)} F(t, d) d t}, \frac{c^{2}}{2 M^{2} \int_{0}^{T} e^{G(t)} \max _{|s| \leq c} F(t, s) d t}\right)$, problem (1.1) has at least three classical solutions.

Proof For all $t \in[0, T]$, we choose $u_{0}(t)=0, \bar{u}(t)=d$. Then, obviously, $u_{0}, \bar{u} \in H, \varphi\left(u_{0}\right)=$ $\phi\left(u_{0}\right)=0, \varphi(\bar{u})=\frac{d^{2}}{2}\left\|e^{G(t)}\right\|_{L^{1}}+\sum_{i=1}^{p} e^{G\left(t_{i}\right)} \int_{0}^{d} I_{i}(t) d t$, and $\phi(\bar{u})=\int_{0}^{T} e^{G(t)} F(t, d) d t$.

Set $r=\frac{c^{2}}{2 M^{2}}$, by simple calculations, we get

$$
\begin{aligned}
\left(r-\varphi\left(u_{0}\right)\right) \frac{\phi(\bar{u})}{\varphi(\bar{u})-\varphi\left(u_{0}\right)} & =\frac{c^{2}}{2 M^{2}} \frac{\int_{0}^{T} e^{G(t)} F(t, d) d t}{\frac{d^{2}}{2}\left\|e^{G(t)}\right\|_{L^{1}}+\sum_{i=1}^{p} e^{G\left(t_{i}\right)} \int_{0}^{d} I_{i}(t) d t} \\
& =\frac{c^{2}}{M^{2}} \frac{\int_{0}^{T} e^{G(t)} F(t, d) d t}{d^{2}\left\|e^{G(t)}\right\|_{L^{1}}+2 \sum_{i=1}^{p} e^{G\left(t_{i}\right)} \int_{0}^{d} I_{i}(t) d t}
\end{aligned}
$$

and combining with $\left(H_{3}\right)$, we obtain $\varphi\left(u_{0}\right)<r<\varphi(\bar{u})$.

For all $u \in H$ satisfying $\varphi(u) \leq r$, combining with (2.1), one has $\|u\|_{H} \leq \sqrt{2 r}$, thus $\|u\|_{\infty} \leq M\|u\|_{H} \leq M \sqrt{2 r}=c$. Therefore,

$$
\sup _{\varphi(x) \leq r} \phi(x) \leq \int_{0}^{T} e^{G(t)} \max _{|s| \leq c} F(t, s) d t<+\infty .
$$


Taking in account of (3.1), (3.2) and $\left(H_{4}\right)$, we have that condition (i) in Theorem 2.1 is satisfied.

Let $T_{n}=\left\{t \in[0, T]:|u(t)|>C_{0}\right\}$. Then from $\left(H_{2}\right)$, there is $C_{1}>0$ such that for all $|u|>C_{0}$ and $t \in[0, T]$,

$$
\begin{aligned}
\varphi(u)-\lambda \phi(u)= & \frac{1}{2}\|u\|_{H}^{2}+\sum_{i=1}^{p} e^{G\left(t_{i}\right)} \int_{0}^{u\left(t_{i}\right)} I_{i}(t) d t-\lambda \int_{0}^{T} e^{G(t)} F(t, u(t)) d t \\
= & \frac{1}{2}\|u\|_{H}^{2}+\sum_{i=1}^{p} e^{G\left(t_{i}\right)} \int_{0}^{u\left(t_{i}\right)} I_{i}(t) d t \\
& -\lambda\left(\int_{T_{n}} e^{G(t)} F(t, u(t)) d t+\int_{[0, T] \backslash T_{n}} e^{G(t)} F(t, u(t)) d t\right) \\
\geq & \frac{1}{2}\|u\|_{H}^{2}-\lambda \beta\|b\|_{L^{1}}\left(a+M^{l}\|u\|_{H}{ }^{l}\right)-\lambda \beta C_{1} .
\end{aligned}
$$

Taking account of $l<2$, one has $\lim _{\|u\|_{H} \rightarrow+\infty}(\varphi(u)-\lambda \phi(u))=+\infty$ for any $\lambda \in \Lambda_{r}$, so condition (ii) in Theorem 2.1 is also satisfied. Hence, combining with Lemmas 2.5 and 2.6, we get the conclusion.

Particularly, we consider $f(t, u)=v(t) h(u)$, where $v \in L^{1}[0, T], h \in C(\mathbb{R}, \mathbb{R})$. Letting $H(u)=\int_{0}^{u} h(s) d s$, we get the following result.

Theorem 3.2 Suppose that $\left(H_{1}\right)$ and $\left(H_{3}\right)$ hold, in addition, assume that

$\left(H_{2}\right)^{\prime}$ there exist positive constants $C_{0}, a, l$ with $l<2$, and a function $b \in L^{1}[0, T]$ such that for all $|u|>C_{0}$ and $t \in[0, T]$

$$
|v(t) H(u)| \leq b(t)\left(a+|u|^{l}\right)
$$

$\left(H_{4}\right)^{\prime}$ there exist $c, d>0$ such that $\frac{M^{2} \max _{|s| \leq c} H(s)\|v(t)\|_{L_{1}}}{c^{2}}<\frac{H(d)\left\|e^{G(t)} v(t)\right\|_{L^{1}}}{d^{2}\left\|e^{G(t)}\right\|_{L^{1}}+2 \sum_{i=1}^{p} e^{G\left(t_{i}\right)} \int_{0}^{d} I_{i}(t) d t}$ hold, then for any $\lambda \in\left(\frac{d^{2}\left\|e^{G(t)}\right\|_{L^{1}}+2 \sum_{i=1}^{p} e^{G\left(t_{i}\right)} \int_{0}^{d} I_{i}(t) d t}{2 H(d)\left\|e^{G(t)} v(t)\right\|_{L^{1}}}, \frac{c^{2}}{2 M^{2} \max _{|s| \leq c} H(s)\|v(t)\|_{L_{1}}}\right)$, problem (1.1) has at least three classical solutions.

Example 3.3 Taking $T=1, t_{1}=\frac{1}{2}, g(t)=-1, f(t, u)=e^{-t} u^{\frac{1}{4}}, I_{1}(u)=u$. Consider the problem

$$
\left\{\begin{array}{l}
-u^{\prime \prime}(t)+u(t)-u^{\prime}(t)=\lambda e^{-t} u^{\frac{1}{4}}, \quad t \in[0,1] \backslash\left\{\frac{1}{2}\right\} \\
\Delta u^{\prime}\left(\frac{1}{2}\right)=u\left(\frac{1}{2}\right), \\
u(0)=u(1)=0 .
\end{array}\right.
$$

We choose $d=1$ and two positive constants $c$ and $M$ such that $\frac{c^{2}}{M^{2}}<3.366$, by direct calculations and applying Theorem 3.1 or Theorem 3.2 , for any $\lambda \in\left(2.103, \frac{5 e c^{\frac{3}{4}}}{8 M^{2}(e-1)}\right)$ problem (3.3) has at least three classical solutions.

\section{Conclusion}

We get the existence of at least three classical solutions for nonlinear impulsive problems with a parameter. Our approach relies on variational methods, more specifically on a three 
critical points theorem by Bonanno and Marano. We show the results by neither adding superlinear or local sublinear assumptions on nonlinear terms at zero nor adding superlinear assumptions on the nonlinearity at infinity.

\section{Acknowledgements}

The authors wish to thank anonymous referees for their valuable suggestions.

\section{Funding}

Supported by the Natural Science Foundation of Shandong Province (ZR2017MA048, ZR2018MG002), the Humanities and Social Sciences Project of the Ministry Education of China (16YJC630070, 19YJA910002), the Taishan Scholars Program of Shandong Province (tsqn20161041), National Natural Science Foundation of China (11571197, 11301303), the Fostering Project of Dominant Discipline and Talent Team of Shandong Province Higher Education Institutions (1716009), Research Funds for Joint Innovative Center for Safe and Effective Mining Technology and Equipment of Coal Resources by Shandong Province and SDUST Research Fund (2014TDJH102).

\section{Availability of data and materials}

Not applicable.

\section{Ethics approval and consent to participate}

All authors contributed to each part of this study equally and declare that they have no competing interests.

\section{Competing interests}

The authors declare that they have no competing interests.

\section{Authors' contributions}

All authors contributed equally to the writing of this paper. The authors read and approved the final manuscript.

\section{Author details}

'School of Mathematics and Quantitative Economics, Shandong University of Finance and Economics, Jinan, P.R. China. ${ }^{2}$ School of Mathematical Sciences, Qufu Normal University, Qufu, P.R. China. ${ }^{3}$ School of Insurance, Shandong University of Finance and Economics, Jinan, P.R. China. ${ }^{4}$ College of Mathematics and Systems Science, Shandong University of Science and Technology, Qingdao, P.R. China.

\section{Publisher's Note}

Springer Nature remains neutral with regard to jurisdictional claims in published maps and institutional affiliations.

Received: 26 December 2018 Accepted: 28 July 2019 Published online: 05 August 2019

\section{References}

1. Nieto, J., O'Regan, D.: Variational approach to impulsive differential equations. Nonlinear Anal. 10, 680-690 (2009)

2. Nieto, J.: Variational formulation of a damped Dirichlet impulsive problem. Appl. Math. Lett. 23, 940-942 (2010)

3. Liu, J., Zhao, Z.Q.: An application of variational methods to second order impulsive differential equation with derivative dependence. Electron. J. Differ. Equ. 2014, 62 (2014)

4. Liu, J., Zhao, Z.Q.: Multiple solutions for impulsive problems with non-autonomous perturbations. Appl. Math. Lett. 64, 143-149 (2017)

5. Samoilenko, A.M., Perestyuk, N.A.: Impulsive Differential Equations. World Scientific, Singapore (1995)

6. Sun, J., Chen, H., Nieto, J., Otero-Novoa, M.: Multiplicity of solutions for perturbed second-order Hamiltonian systems with impulsive effects. Nonlinear Anal. 72, 4575-4586 (2010)

7. Tian, Y., Ge, W.: Applications of variational methods to boundary value problem for impulsive differential equations. Proc. Edinb. Math. Soc. 51, 509-527 (2008)

8. Zhang, T.Q., Meng, X.Z., Song, Y., Zhang, T.H.: A stage-structured predator-prey SI model with disease in the prey and impulsive effects. Math. Model. Anal. 4, 505-528 (2013)

9. Zhang, T.Q., Meng, X.Z., Zhang, T.H., Song, Y.: Global dynamics for a new high-dimensional SIR model with distributed delay. Appl. Math. Comput. 218, 11806-11819 (2012)

10. Liu, K.Y., Zhang, T.Q., Chen, L.S.: State-dependent pulse vaccination and therapeutic strategy in an SI epidemic model with nonlinear incidence rate. Comput. Math. Methods Med. 2019, Article ID 3859815 (2019)

11. Zhang, T.Q., Ma, W.B., Meng, X.Z.: Global dynamics of a delayed chemostat model with harvest by impulsive flocculant input. Adv. Differ. Equ. 2017, 115 (2017)

12. Liu, J., Zhao, Z.Q., Zhang, T.Q.: Multiple solutions to damped Hamiltonian systems with impulsive effects. Appl. Math. Lett. 91, 173-180 (2019)

13. Candito, P., Carl, S., Livrea, R.: Multiple solutions for quasilinear elliptic problems via critical points in open sublevels and truncation principles. J. Math. Anal. Appl. 395, 156-163 (2012)

14. Candito, P., Carl, S., Livrea, R.: Critical points in open sublevels and multiple solutions for parameter-depending quasilinear elliptic equations. Adv. Differ. Equ. 19, 11/12 (2014)

15. Candito, P., Carl, S., Livrea, R.: Variational versus pseudomonotone operator approach in parameter-dependent nonlinear elliptic problems. Dyn. Syst. Appl. 22, 397-410 (2013)

16. Bonanno, G., Marano, S.A.: On the structure of the critical set of non-differentiable functions with a weak compactness condition. Appl. Anal. 89, 1-10 (2010) 
17. Bonanno, G., Riccobono, G.: Multiplicity results for Sturm-Liouville boundary value problems. Appl. Math. Comput. 210, 294-297 (2009)

18. Mawhin, J., Willem, M.: Critical Point Theory and Hamiltonian System. Springer, Berlin (1989)

19. Zeidler, E.: Nonlinear Functional Analysis and Its Applications. Springer, Berlin (1990)

Submit your manuscript to a SpringerOpen ${ }^{\circ}$ journal and benefit from:

- Convenient online submission

- Rigorous peer review

- Open access: articles freely available online

- High visibility within the field

- Retaining the copyright to your article

Submit your next manuscript at $\gg$ springeropen.com 